ARTICLE

DOI: $10.1038 /$ s41467-017-00588-3

OPEN

\title{
Dipole-field-assisted charge extraction in metal-perovskite-metal back-contact solar cells
}

Xiongfeng Lin ${ }^{1,10}$, Askhat N. Jumabekov², Niraj N. Lal ${ }^{1,2}$, Alexander R. Pascoe ${ }^{1}$, Daniel E. Gómez ${ }^{2,3}$, Noel W. Duffy (1) 4, Anthony S.R. Chesman 2,5, Kallista Sears' ${ }^{2}$, Maxime Fournier ${ }^{6}$, Yupeng Zhang (1) 1,7, Qiaoliang Bao (iD) 1, Yi-Bing Cheng 1,5, Leone Spiccia ${ }^{6}$ \& Udo Bach²,5,8,9

Hybrid organic-inorganic halide perovskites are low-cost solution-processable solar cell materials with photovoltaic properties that rival those of crystalline silicon. The perovskite films are typically sandwiched between thin layers of hole and electron transport materials, which efficiently extract photogenerated charges. This affords high-energy conversion efficiencies but results in significant performance and fabrication challenges. Herein we present a simple charge transport layer-free perovskite solar cell, comprising only a perovskite layer with two interdigitated gold back-contacts. Charge extraction is achieved via self-assembled monolayers and their associated dipole fields at the metal-perovskite interface. Photovoltages of $\sim 600 \mathrm{mV}$ generated by self-assembled molecular monolayer modified perovskite solar cells are equivalent to the built-in potential generated by individual dipole layers. Efficient charge extraction results in photocurrents of up to $12.1 \mathrm{~mA} \mathrm{~cm}^{-2}$ under simulated sunlight, despite a large electrode spacing.

\footnotetext{
${ }^{1}$ Department of Materials Science \& Engineering, Monash University, Clayton, VIC 3800, Australia. ${ }^{2}$ CSIRO Manufacturing, Clayton, VIC 3168, Australia ${ }^{3}$ Plasmonics \& Photochemistry Laboratory, RMIT University, Melbourne, VIC 3000, Australia. ${ }^{4}$ CSIRO Energy, Clayton, VIC 3168 , Australia. ${ }^{5}$ ARC Centre of Excellence in Exciton Science, Monash University, Clayton, VIC 3800, Australia. ${ }^{6}$ School of Chemistry and ARC Centre of Excellence in Electromaterials Science, Monash University, Clayton, VIC 3800, Australia. ${ }^{7}$ College of Electronic Science and Technology, Shenzhen University, Shenzhen 518000, China. ${ }^{8}$ Melbourne Centre for Nanofabrication, Clayton, VIC 3800, Australia. ${ }^{9}$ Department of Chemical Engineering, Monash University, Clayton, VIC 3800 , Australia. ${ }^{10}$ Present address: Department of Chemical Engineering, Monash University, Clayton, VIC 3800, Australia. Xiongfeng Lin and Askhat N. Jumabekov contributed equally to this work. Correspondence and requests for materials should be addressed to U.B. (email: udo.bach@monash.edu)
} 
ybrid organic-inorganic halide perovskites are amongst the most promising materials for photovoltaic applications ${ }^{1-4}$. On the basis of earth abundant, solutionprocessable, low-cost materials, this subclass of perovskite compounds exhibits attributes that challenge silicon's predominance in photovoltaics. Efficient charge separation in perovskite solar cells (PSCs) is typically achieved by carrier selective charge transport layers (CTLs), which exist as either conventional transport materials or ultrathin work function modifiers ${ }^{5}$. The fabrication of pinhole and defect free multi-layer structures is challenging, especially for moisture-sensitive solution-processable materials. Furthermore, key challenges facing the field of PSCs, such as enhanced recombination, poor stability, and hysteresis during current-voltage scans, are linked to carrier selective CTLs and their respective CTL-perovskite interfaces ${ }^{6-8}$.

The photovoltaic properties of CTL-free PSCs, in which metals or graphene directly contact the perovskite to create Schottky junction solar cells ${ }^{9-14}$, depend strongly on the built-in potential established by the work function difference of the two contacts. These differences can be temporarily induced in gold-perovskitegold solar cells by applying a large external bias (greater than $2 \mathrm{~V}$ ) across the symmetric contacts (poling), resulting in migration and accumulation of $\mathrm{CH}_{3} \mathrm{NH}_{3}{ }^{+}$and $\mathrm{I}^{-}$ions at the respective contacts $^{13-20}$. However, poling is not sufficient to provide a stabilized power output.

Persistent work function modification of metals and semiconductors can be induced by self-assembled monolayers (SAMs) featuring oriented molecules with permanent dipole moments $^{21-24}$. The built-in potential of heterojunctions can be adjusted accordingly by placing these SAMs directly at the junction interface. This concept has been demonstrated successfully in organic light-emitting-diodes and organic field-effect transistors, but not explicitly within solar cells ${ }^{25-27}$.

Herein we present a simple gold-perovskite-gold back-contact PSC (bc-PSCs) with a built-in potential and photovoltaic response owing exclusively to dipole SAMs present at the gold-perovskite interfaces. An interdigitated gold microelectrode array (IDA) on glass forms the back-contact of the solar cell. The SAMs are formed by two para-substituted thiolbenzene derivatives with opposing molecular dipoles. Both molecules are insulators and do not play an active role in the charge transport process. We present a method to selectively modify the two sets of IDA microelectrodes, termed "a" and " $b$ ", with the SAM compounds. This modification induces a work function difference between the two microelectrodes of up to $600 \mathrm{mV}$, as observed through Kelvin probe force microscopy (KPFM). Bc-PSCs are fabricated by spin-coating a perovskite precursor solution onto the SAM-modified IDA in a single step. The opencircuit potential $\left(V_{\mathrm{OC}}\right)$ of these bc-PSCs is shown to be equivalent to the work function difference induced by the SAM modification $\left(\phi_{\mathrm{m}}^{(\mathrm{b})}-\phi_{\mathrm{m}}^{(\mathrm{a})}\right)$. Photocurrent mapping measurements reveal near uniform (less than 25\%) charge collection across the cell, giving rise to short-circuit photocurrent densities $\left(J_{\mathrm{SC}}\right)$ of up to $12.1 \mathrm{~mA} \mathrm{~cm}^{-2}$ despite the large $6.5 \mu \mathrm{m}$ centre-to-centre (distance from the centre of electrode "a" to the centre of adjacent electrode "b") and $4.3 \mu \mathrm{m}$ edge-to-edge (distance from the edge of electrode "a" to the edge of adjacent electrode " $b$ ") electrode distances.

\section{Results}

Work function modification of IDA. The detailed steps used to fabricate the modified interdigitated microelectrode arrays are given in the "Methods" section (Supplementary Fig. 1). In brief, the pristine gold IDA (Supplementary Fig. 2a) is immersed into an OMeTP solution, resulting in the formation of OMeTP SAMs on both microelectrodes "a" and " $b$ ". The OMeTP SAM on electrode " $b$ " is subsequently desorbed electrochemically. The complete IDA is then exposed briefly to a solution of ClTP in order to form the respective SAM on microelectrode " $b$ ".

Kelvin-probe-force-microscopy (KPFM) was used to monitor the surface potential changes of the gold IDAs after each fabrication step. KPFM allows for the direct mapping of the contact potential difference between the AFM tip and the sample surface $\left(\mathrm{CPD}=\phi_{\text {sample }}-\phi_{\text {tip }}\right)$, hence, quantifying the work function difference $\left(\phi_{\mathrm{m}}^{(\mathrm{b})}-\phi_{\mathrm{m}}^{(\mathrm{a})}\right)$ between electrodes "b" and "a". Figure la shows the KPFM image of an IDA section (Fig. 1b) prior to SAM modification. All fingers initially have a similar $\mathrm{CPD}$ of approximately $+100 \mathrm{mV}$. Figure $1 \mathrm{c}$ shows the KPFM image after OMeTP adsorption and electrochemical desorption from electrode " $b$ ". The formation of the OMeTP SAM on electrode "a" is confirmed indirectly by the $400 \mathrm{mV} \mathrm{CPD}$ increase relative to the bare electrode, while electrode " $b$ " increases by $\sim 100 \mathrm{meV}$ due to the incomplete desorption of the OMeTP SAM. The subsequent exposure to the C1TP solution further increases this work function difference to $550-580 \mathrm{meV}$ (Fig. 1e). These $\mathrm{CPD}$ results agree well with photoelectron spectroscopy in air a

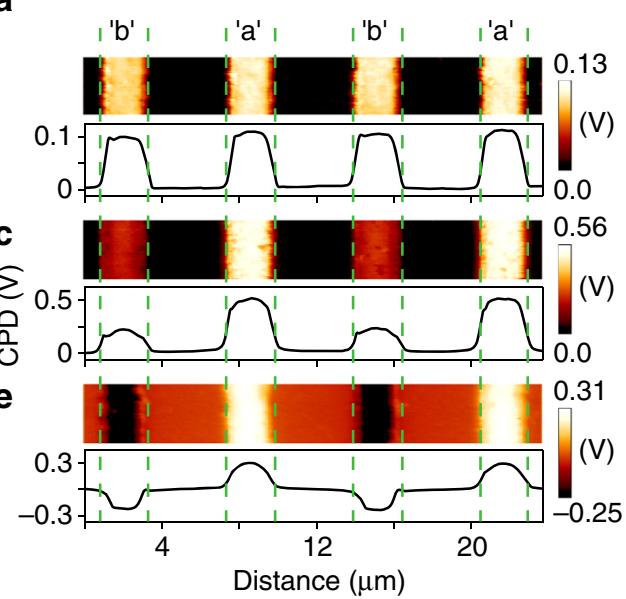

b

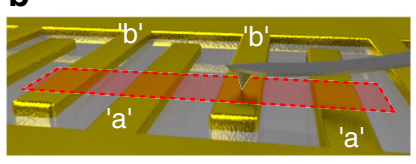

d

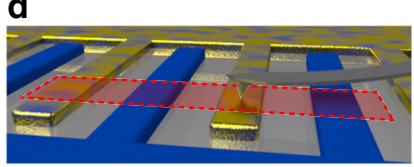

f

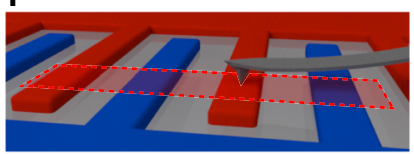

Fig. 1 Kelvin-probe-force-microscopy of an interdigitated gold microelectrode array during molecular modification. Contact potential difference (CPD) maps across sets of interdigitated fingers and illustrations of the KPFM imaging experiment for an unmodified IDA $\mathbf{a}+\mathbf{b}$, an IDA after exposure to 4methoxythiophenol (OMeTP) and its subsequent electrochemical desorption from electrode " $b$ " $\mathbf{c}+\mathbf{d}$, and an IDA after the final modification step with 4chlorothiophenol (CITP) e +f 
a

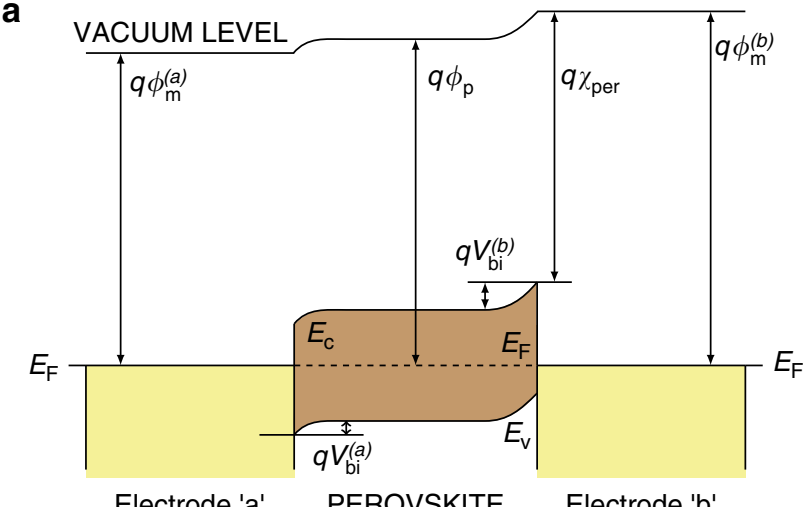

b

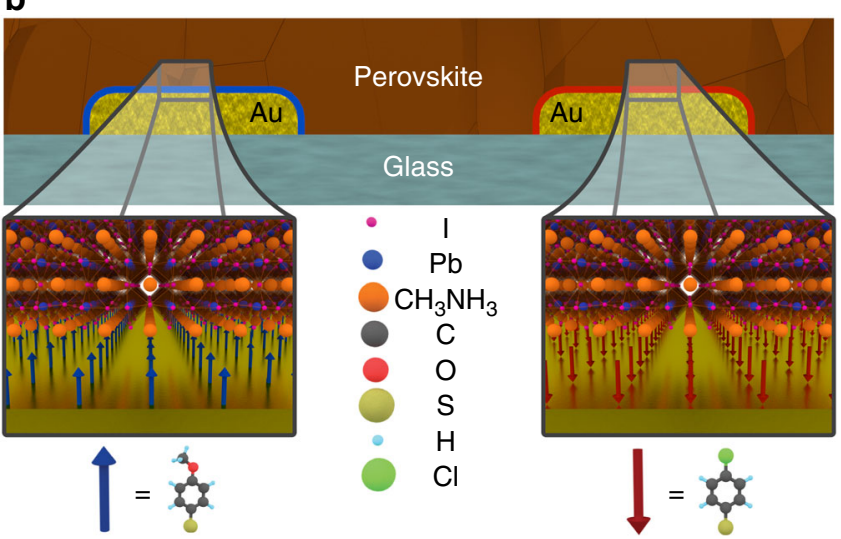

Fig. 2 Back-contact metal-perovskite-metal solar cells. a Schematic energy band diagram of a metal-perovskite-metal solar cell at thermal equilibrium in the dark. The work function of the methylammonium lead iodide $\left(\mathrm{MAPbl}_{3}\right)$ perovskite $\left(q \phi_{\mathrm{p}}\right)$ is situated between those of the metal electrodes "a" $\left(q \phi_{\mathrm{m}}^{(\mathrm{a})}\right)$ and "b" $\left(q \phi_{\mathrm{m}}^{(\mathrm{b})}\right)$. The overall built-in potential of the solar cell is equal to the sum of the built-in potentials at the metalperovskite contacts. $q \chi_{\text {per }}$ corresponds to the electron affinity of $\mathrm{MAPbl}_{3}$. b Cross-section diagram of a dipole self-assembled monolayer (SAM) modified back-contact gold-perovskite-gold solar cell. Electrode "a" (anode, left) is modified with a molecular monolayer of 4-methoxythiophenol (OMeTP) with a molecular dipole of $-2.67 \mathrm{D}$. Electrode " $b$ " (cathode, right) is modified with a monolayer of 4-chlorothiophenol (CITP) with a molecular dipole of $+1.41 \mathrm{D}$

(PESA) measurements on SAM-modified gold films (Supplementary Fig. 3). CPD, PESA and Raman spectroscopy measurements (Supplementary Fig. 4) provide evidence for the incomplete electrochemical desorption of OMeTP from electrode "b" (Fig. 1d) and some co-adsorption of ClTP on electrode "a" via thiolexchange reactions during the final modification step (Fig. 1f). These measurements indicate that stronger built-in potentials could be obtained by avoiding these cross-contamination processes.

Performance of dipole modified bc-PSCs. We fabricated "a"- $\mathrm{MAPbI}_{3}$-"b" bc-PSCs by spin-coating a perovskite precursor solution containing $\mathrm{PbI}_{2}$ and $\mathrm{CH}_{3} \mathrm{NH}_{3} \mathrm{I}$ in dimethyl sulfoxide (DMSO) and N-methyl-2-pyrrolidone (NMP) (v/v 7:3) onto the OMeTP and CITP SAM-modified IDAs (see "Methods" section for details). Figure 2a shows an energy band diagram for an "a"-MAPbI ${ }_{3}$ " b" metal-perovskite-metal solar cell (see Supplementary Fig. 5 for details). The illustration of the cross section of the "a"-MAPbI ${ }_{3}$ "b" solar cell device is shown in Fig. 2b.

The photovoltaic performance was measured via photocurrent density versus voltage $(J-V)$ curves under simulated sunlight (AM
$1.5 \mathrm{G} ; 1000 \mathrm{~W} \mathrm{~m}^{-2}$ ). The active area of the device is defined by the interdigitated area of the gold electrodes, which is $2 \times 2 \mathrm{~mm}$ (Supplementary Fig. 2). Figure 3a shows $J-V$ curves for a typical device recorded in forward scan mode $\left(0 \mathrm{~V} \rightarrow V_{\mathrm{OC}}\right)$ and reverse scan mode $\left(V_{\mathrm{OC}} \rightarrow 0 \mathrm{~V}\right)$. The scans reveal negligible hysteresis and a $V_{\mathrm{OC}}$ of 560 and $570 \mathrm{mV}$ for forward and reverse scan modes, respectively. This is similar to the work function difference of $550-580 \mathrm{meV}$ measured for the SAM-modified back-contact plane. A strong correlation between $V_{\mathrm{OC}}$ and the work function difference between the anode and cathode was also observed for bc-PSCs where only one of the two electrodes was modified with either a OMeTP or ClTP SAM (Supplementary Figs. $6,7 \mathrm{~b}$ ). This finding suggests that larger $V_{\mathrm{OC}}$ values can be achieved using SAMs that further increase the work function difference between the contacts. Bc-PSCs fabricated without contact modification produced devices with a $V_{\mathrm{OC}}$ of less than $100 \mathrm{mV}$, confirming that the built-in potential was fundamentally a result of the SAM modification. The recorded $J_{S C}$ values of 11.4 and $11.3 \mathrm{~mA} \mathrm{~cm}^{-2}$ for forward and reverse scan modes, respectively, are high, considering the large centre-to-centre $(6.5 \mu \mathrm{m})$ and edge-to-edge $(4.3 \mu \mathrm{m})$ electrode distances. With fill factors (FF) of $40.5 \%$ and $40.1 \%$, power conversion efficiencies (PCE) of $2.59 \%$ and $2.57 \%$ were obtained for the unencapsulated device in forward and reverse scan modes, respectively (see Fig. 3a).

The $J-V$ curves after encapsulation (Supplementary Fig. 2d) show a slight decrease in the photovoltaic parameters for the cell (Fig. 3a). The $V_{\mathrm{OC}}, J_{\mathrm{SC}}$ and $F F$ of the encapsulated device are 0.55 $\mathrm{V}, 9.73 \mathrm{~mA} \mathrm{~cm}^{-2}$ and $36.7 \%$, respectively, with a PCE of $1.96 \%$ for reverse scan mode. In forward scan mode, the encapsulated device shows $0.54 \mathrm{~V}, 9.87 \mathrm{~mA} \mathrm{~cm}^{-2}$, and $36.7 \%$ for the $V_{\mathrm{OC}}, J_{\mathrm{SC}}$ and $F F$, respectively, resulting in a PCE of $1.96 \%$. Slight decreases of photovoltaic performance may originate from reflective losses due to the introduction of the glass cover over the perovskite layer, as well as overall device degradation due to the encapsulation process. The external quantum efficiency (EQE) of an encapsulated device was seen to be largely independent of wavelength in the $350-900 \mathrm{~nm}$ range, with a value in excess of $33 \%$. The determined AM $1.5 \mathrm{G}$ short-circuit current obtained from the EQE spectrum (dashed curve in Fig. 3b) of $9 \mathrm{~mA} \mathrm{~cm}^{-2}$ is in agreement with the $J_{\mathrm{SC}}$ value of $9.73 \mathrm{~mA} \mathrm{~cm}^{-2}$ derived from the $J-V$ characteristics of the encapsulated device (Fig. 3a). Monitoring the performance of SAM modified bc-PSCs at the maximum power point (MPP) as a function of time revealed that the solar cells exhibit a stable power output $\left(P_{\mathrm{MPP}}\right)$ (Fig. $3 \mathrm{c}$ and d), with a value of around $2.3 \%$. The highest performing bc-PSC fabricated in this study showed an efficiency of $3.11 \%$ (Supplementary Figs. $7 \mathrm{a}, 8)$ with the $J_{\mathrm{SC}}$ value of $12.1 \mathrm{~mA} \mathrm{~cm}^{-2}$. For comparison, $J-V$ measurements on unmodified gold-perovskite-gold $\left(\mathrm{Au}-\mathrm{MAPb}_{3}-\mathrm{Au}\right)$ devices showed no photovoltaic effect (Supplementary Fig. $7 \mathrm{~b})^{13}, 15,16$. Poling induced by a $2 \mathrm{~V}$ external bias for $80 \mathrm{~s}$ (Fig. $3 \mathrm{c}, \mathrm{d}$ ) initially resulted in a $P_{\mathrm{MPP}}$ of $0.13 \mathrm{~mW} \mathrm{~cm}^{-2}$, however, this photovoltaic effect was momentary, with the $P_{\mathrm{MPP}}$ dropping by $\sim 90 \%$ over the 150 s observation period.

Photocurrent and photoluminescence mapping. Photocurrent (PC) and photoluminescence (PL) maps were recorded simultaneously using a confocal laser beam scan across the solar cell's active area (Fig. 4a, b, see Methods for details). The PC profile features photocurrent minima above both collection electrodes and maxima within the electrode gaps, consistent with previous reports of balanced electron and hole diffusion lengths in $\mathrm{MAPbI}_{3}{ }^{28}$. For charges generated at the mid-point between "a" and " $b$ " electrodes, the minimum travel distance for collection is $\sim 2 \mu \mathrm{m}$, while one of the two carriers is required to travel at least $5.3 \mu \mathrm{m}$ when generated directly above an electrode. The PC 

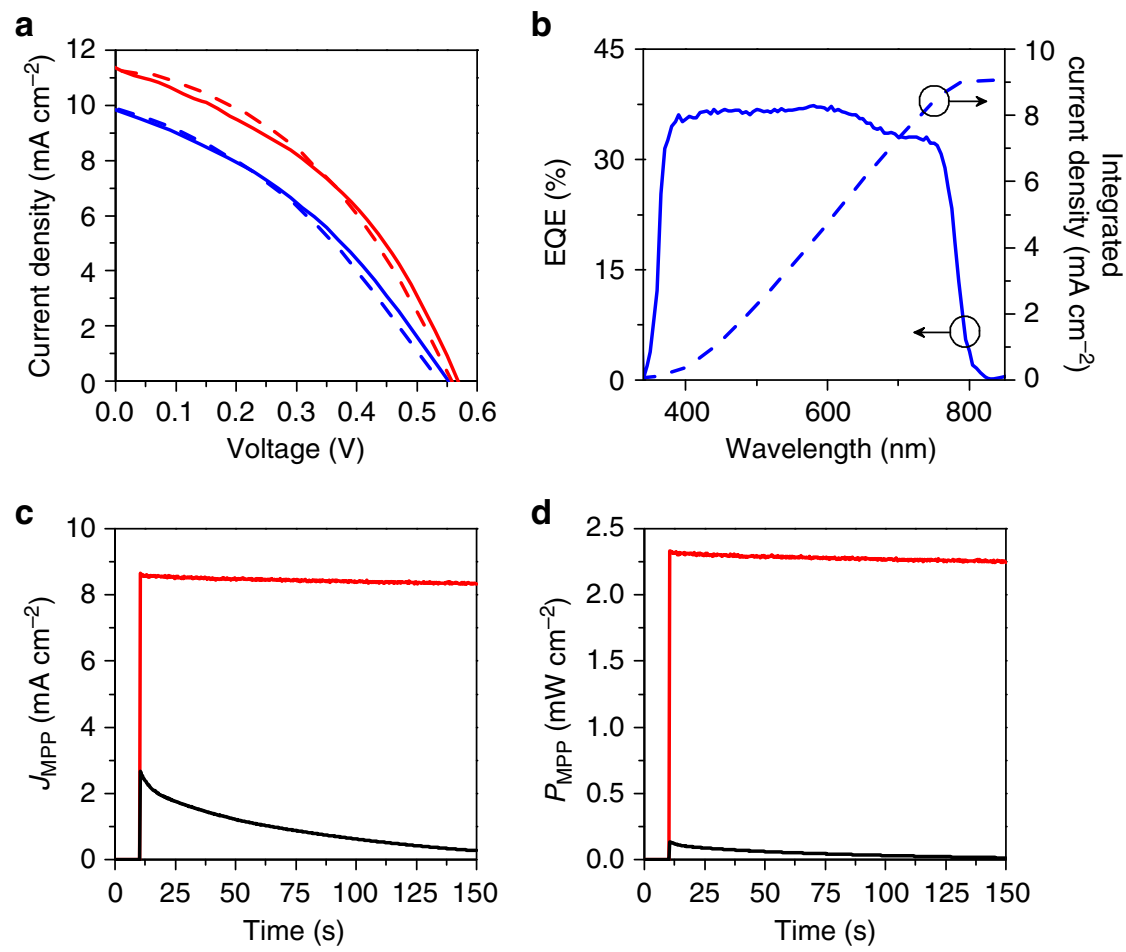

Fig. 3 Photovoltaic properties of self-assembled monolayer modified back-contact perovskite solar cells. a $J-V$ curves of a dipole-modified gold-perovskitegold bc-PSC measured under standard solar irradiation (AM 1.5 G, $1000 \mathrm{~W} \mathrm{~m}^{-2}$ ), reverse scan (solid blue, encapsulated; solid red, unencapsulated) and forward scan (dashed blue, encapsulated, dashed red, unencapsulated). b Spectral response (External quantum efficiency - EQE, solid line) and the corresponding integrated short-circuit current density (dashed line) of a SAM-modified PSC. c Time evolution of the maximum power point photocurrent density ( $J_{\text {MPP }}$ ) of a SAM-modified (red) and poled, unmodified bc-PSC (black) and their power output $\mathbf{d}$

profile shows the overall variation in EQE is $<25 \%$ across the entire cell, indicating excellent charge-carrier extraction. Variations in the perovskite film thickness as source of PC or PL fluctuations can be excluded, with $>90 \%$ of the scanning laser light absorbed within the top $200 \mathrm{~nm}$ of the $\mathrm{MAPbI}_{3}$ film.

The PL map shows luminescence maxima for excitation above OMeTP modified "a" electrodes and minima above CITB modified "b"-electrodes. The luminescence intensity above both electrodes differs by about two orders of magnitude (Supplementary Fig. 9). The PL appears mostly featureless above electrodes " $b$ " but shows distinct grain-like features near the centre of each electrode "a" and inside the electrode gaps. This is consistent with the AFM topography image (Fig. $4 \mathrm{~d}, \mathrm{e}$ ) that reveals large multimicron-sized $\mathrm{MAPbI}_{3}$ crystalline regions along electrode " $\mathrm{b}$ ", as well as a pronounced microgap located at the centre of each "a" electrode. This provides some indication that the $\mathrm{MAPbI}_{3}$ film morphology appears to be the major contributing factor to the PL variations observed. Isolated, highly luminescent $\mathrm{MAPbI}_{3}$ crystalline regions within this microgap are a likely source for the strongly luminescent features observed in the PL map. The observed topography patterns result from the specific bc-PSC architecture and the different wetting properties of the OMeTPand ClTB-functionalized electrodes affecting the crystallization and film formation process (Supplementary Fig. 10), as confirmed by contact angle measurements on bare and SAM-modified gold films, in which the OMeTP-modified gold films showed significantly better wetting than the bare or ClTB-modified gold films (Supplementary Fig. 11).

Modelling of theoretical PCE limit and charge collection efficiency. A number of simulations were carried out in order to gage the performance limit of Schottky junction PSCs and more specifically back-contact Schottky PSCs. First, we estimated the maximum theoretical PCE for a $\mathrm{MAPbI}_{3}$ Schottky junction solar cell to be $25 \%$, based on a thermionic emission model ${ }^{29}$ (Fig. 5a). This efficiency limit is expected for an ideal Schottky junction $\mathrm{MAPbI}_{3}$ solar cell in which the junction barrier height equals the value of its bandgap of $1.6 \mathrm{eV}$. Similar efficiency limits of $25-27 \%$ have recently been derived for PSCs with conventional $p-i-n$ heterojunction structures ${ }^{30}$. For the OMeTP and ClTP-modified bc-PSCs presented here we calculated a barrier height of $1.2 \mathrm{eV}$ and a corresponding maximum theoretical efficiency limit of $15 \%$ (see "Methods" section for details). Further transfer matrix optical modelling of the "a"-MAPbI- "b" device yielded an upper limit of $19.9 \mathrm{~mA} \mathrm{~cm}^{-2}$ as the maximum theoretical $J_{\mathrm{SC}}$ under AM1.5 G solar irradiation for the bc-PSCs reported here. This estimation is simply based on the overall optical light harvesting efficiency of the device (see Supplementary Fig. 12 and "Methods" section for details) ${ }^{31}$.

Ultimately, the charge collection efficiency of bc-PSCs is dependent on the relationship between electrode separation distance and the charge carrier diffusion length $L_{\mathrm{d}}$. Fig. 5b depicts the collection efficiency (normalized $J_{\mathrm{SC}}$ ) as a function of $L_{\mathrm{d}}$ and electrode separation distance, and is calculated based on the formalism of Taretto et al. ${ }^{32}$ with a built in voltage of $0.6 \mathrm{~V}$ and balanced diffusion lengths. The model predicts that charge collection efficiencies close to $100 \%$ for situations in which the electrode spacing is comparable to, or smaller than, the diffusion length. We also used the same formalism by Taretto et al. ${ }^{32}$ to calculate a charge carrier diffusion length of $350 \mathrm{~nm}$ for the encapsulated "a"-MAPbI ${ }_{3}^{-}$"b" bc-PSCs, based on the photocurrent mapping data shown in Fig. $4 c^{32-34}$ (see "Methods" section and Supplementary Fig. 13 for details). On the basis of this value and the geometrical electrode spacing (edge-to-edge) of $4.3 \mu \mathrm{m}$ the model predicts a charge carrier collection efficiency for the current configuration of $\sim 40 \%$ (see dashed lines in Fig. $5 \mathrm{~b}$ ). This is 
a

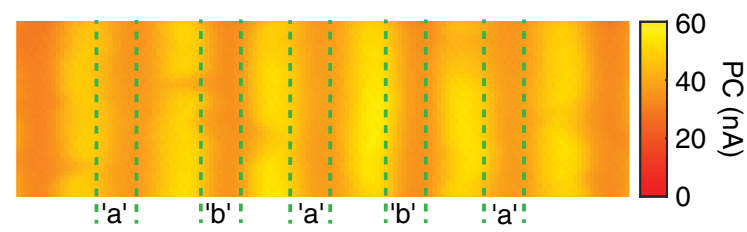

b
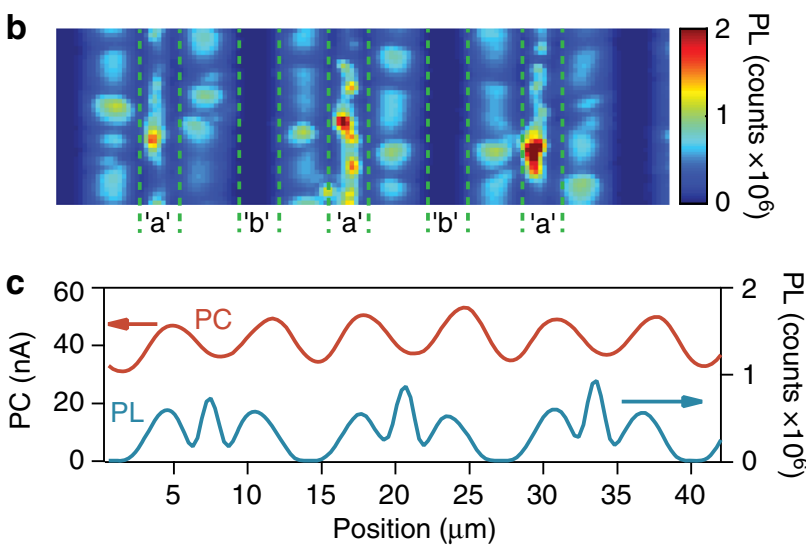

d

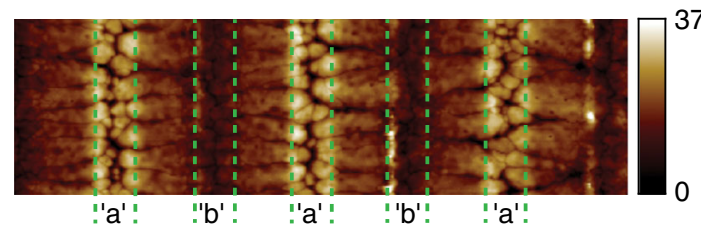

$\frac{1}{\mathbb{D}}$
$\frac{6}{0}$
$\frac{1}{3}$
$\frac{3}{3}$

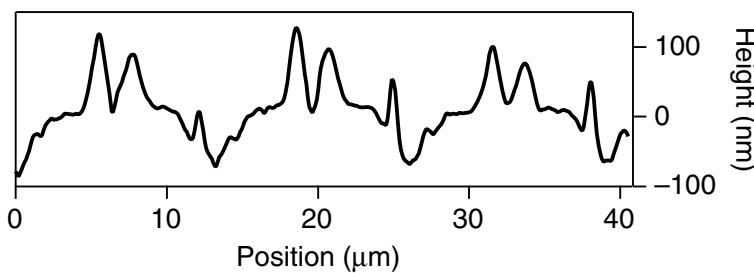

Fig. 4 Spatial photocurrent and photoluminescence mapping of selfassembled monolayer modified back-contact perovskite solar cells. a Spatial PC map of a SAM-modified bc-PSC at short-circuit condition. b Spatial PL map of a SAM-modified bc-PSC at short-circuit condition. c $P C$ and PL profile obtained by averaging the PC and PL maps along the vertical direction. d Atomic-force-microscopy (AFM) topography map of a SAM-modified bc-PSC. e AFM topography map profile of a SAM-modified bc-PSC. The scanned area in all subpanels is $42 \times 12 \mu \mathrm{m}$

in excellent agreement with the EQE data (Fig. 3b), which also shows around $40 \%$ for the same encapsulated "a"- $\mathrm{MAPbI}_{3}$-"b" bc-PSCs at illumination wavelength of $532 \mathrm{~nm}$.

\section{Discussion}

In conclusion, we have presented the first implementation of a photovoltaic device, in which charge extraction is solely achieved by a dipole field generated via molecular monolayers. We expect this general concept to be adaptable to a wide range of absorber, electrode and molecular dipole materials well beyond the capabilities shown here. Through modelling we demonstrated that high-performance solar cell devices can be obtained using the back-contact electrode design with a higher work function asymmetry between the electrodes and optimized electrode spacing. A number of important technical advantages arise from abolishing the need for additional CTLs in concert with backcontact designs. This back-contact design concepts ${ }^{35}$ combined with scalable low-cost nanofabrication techniques ${ }^{36}$ offer the potential for boosting solar cell efficiencies while providing scope for mass-production at low cost.

\section{Methods}

Chemicals. Unless specified otherwise, all materials for perovskite precursor preparation were purchased from either Alfa Aesar or Sigma-Aldrich and used as received. 4-Methoxythiophenol (OMeTP, Sigma-Aldrich, $\geq 98.0 \%$ ) and 4-chlorothiophenol (ClTP, Apollo Scientific Limited) were used without further purification. Reverse osmosis (RO) purified water with a quoted resistivity of $1 \mathrm{M} \Omega$ $\mathrm{cm}$ at $25^{\circ} \mathrm{C}$ was used for all desorption experiments.

Perovskite precursor preparation. Methylammonium iodide, $\mathrm{CH}_{3} \mathrm{NH}_{3} \mathrm{I}$ (MAI), was synthesized according to a previous study ${ }^{37}$. To synthesize MAI, $24 \mathrm{ml}$ methylamine $\left(\mathrm{CH}_{3} \mathrm{NH}_{2}, 33 \%\right.$ in ethanol) was mixed with $10 \mathrm{ml}$ hydroiodic acid (HI, $57 \%$ in water) in $100 \mathrm{ml}$ ethanol. The solvent was removed under vacuum to give a powder of MAI, which was subsequently washed with diethyl ether and used without further purification.

Fabrication of gold interdigitated microelectrode arrays. Initially, $25 \times 25 \mathrm{~mm}$ soda-lime glass substrates were cleaned by sequential sonication steps in acetone and 2-propanol solutions, each performed for $15 \mathrm{~min}$. After drying with compressed air, a $2 \mu \mathrm{m}$ thick photoresist layer was spin coated onto the glass substrates and baked at $110^{\circ} \mathrm{C}$ for $2 \mathrm{~min}$. The substrates were then exposed to UV light through a chrome photo-mask with the desired pattern. In the developmental step, the UV-exposed sections of the photoresist layer were removed by washing the substrate in a developer solution, resulting in a portion of the substrates being protected by the photoresist mask. In the next step, $10 \mathrm{~nm}$ Ti and $60 \mathrm{~nm}$ Au layers were sequentially evaporated onto the substrates using an electronbeam evaporator. Finally, the remainder of the polymer mask was removed by sonicating the substrates in acetone (lift-off process), with six IDA electrodes deposited onto a single substrate (Supplementary Fig. 2a). The yield of the IDA fabrication process was around $98 \%$. The interdigitated area of the electrodes is $2 \times 2 \mathrm{~mm}$, in which the width of the IDA fingers is approximately $2.2 \mu \mathrm{m}$ and the gap between the interdigitated fingers is $\sim 4.3 \mu \mathrm{m}$ (Supplementary Fig. $2 \mathrm{~b}$ ).

Work function modification of gold IDAs with thiophenol-based SAMs. The selective deposition of 4-methoxythiophenol (OMeTP) and 4-chlorothiophenol (ClTP) onto the separate gold components of the IDA requires a stepwise protocol (Supplementary Fig. 1a). Prior to modification, the gold IDA was treated with ozone plasma (Harrick Plasma) for $18 \mathrm{~min}$. For OMeTP-modification, the substrate was immersed in a $20 \mathrm{mM}$ OMeTP in ethanol solution for $24 \mathrm{~h}$ in an inert atmosphere. The OMeTP-modifed IDA was then rinsed with anhydrous ethanol and dried under a nitrogen flow. Desorption of the OMeTP monolayer from one half of the IDA was achieved through an electrochemical process (see below), followed by rinsing of the substrate with RO water and ethanol, and drying with a nitrogen flow. Desorption of the OMeTP monolayer on one half of the IDA was verified by KPFM measurements (see below). A ClTP monolayer was then deposited onto the unmodified part of the IDA by soaking the substrate in a $10 \mathrm{mM}$ ClTP in ethanol solution for $1 \mathrm{~min}$. The OMeTP- (electrode "a") and CITPmodified (electrode "b") IDA was then rinsed with anhydrous ethanol and dried under a nitrogen flow.

Electrochemical treatment for desorption of OMeTP. Desorption of the OMeTP monolayer from one half of the IDA was obtained by using a Bio-Logic VSP instrument configured in bipotentiostat mode (Supplementary Fig. 1) ${ }^{38-40}$. The working electrodes were the two halves of the IDA. The counter-electrode was tin wire and the reference electrode was $\mathrm{Ag} \mid \mathrm{AgCl} / \mathrm{NaCl}(3 \mathrm{M})$ (CH-Instrument). All experiments were carried out in ambient air at $21^{\circ} \mathrm{C}$ in a $0.5 \mathrm{M} \mathrm{KOH}$ aqueous solution. No degassing process was applied to the electrolyte during the desorption procedure.

A potential of $-0.144 \mathrm{~V}$ vs. $\mathrm{Ag} / \mathrm{AgCl}$ (corresponding to the open circuit potential of the modified surface) was constantly maintained for the upper half of the IDA (Supplementary Fig. 1a) in order to prevent any desorption of OMeTP. At the same time, $-1.05 \mathrm{~V}$ vs. $\mathrm{Ag} / \mathrm{AgCl}$ was applied to the lower half of the IDA until the evolution of current over time stagnated over about $200 \mu \mathrm{A}$, corresponding to the capacitive current of an unmodified gold surface (Supplementary Fig. 1b).

Perovskite deposition. $\mathrm{PbI}_{2}$ and MAI were dissolved in a 1:1 molar ratio in a solution of dimethyl sulfoxide (DMSO) and N-methyl-2-pyrrolidone (NMP) (v/v $7: 3$ ) to obtain a $1.74 \mathrm{M}$ perovskite precursor solution. The $\mathrm{MAPbI}_{3}$ thin-films were formed in an inert atmosphere by spin coating the precursor solution onto the substrates using a two-step program (500 rpm for $10 \mathrm{~s}$, then $6000 \mathrm{rpm}$ for $50 \mathrm{~s}$ ). $\mathrm{A} \mathrm{N}_{2}$ gas stream was introduced at the $20 \mathrm{~s}$ mark of the second step, and sustained for a further $20 \mathrm{~s}$. The films were then transferred into a covered glass petri dish containing two droplets $(0.5 \mu \mathrm{l}$ each) of a DMSO/NMP (v/v 7:3) solution. Perovskite films were solvent annealed at $100{ }^{\circ} \mathrm{C}$ inside a closed petri dish for $5 \mathrm{~min}$, and the petri dish was then opened for another $5 \mathrm{~min}$ of annealing. The film thickness on top of the IDA electrodes has an average value of about $340 \mathrm{~nm}$. 

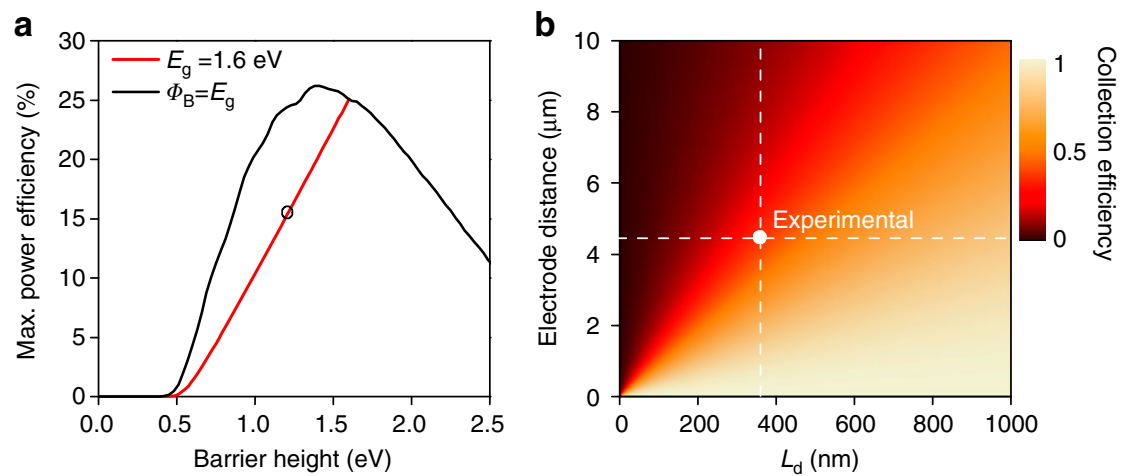

Fig. 5 Modelling of theoretical power conversion efficiency limit and collection efficiency. a Theoretical power conversion efficiency limit (red) as a function of barrier height $\Phi_{B}$, calculated for a Schottky junction $\mathrm{MAPb}_{3}$ back-contact perovskite solar cells (bc-PSCs) under AM1.5 G irradiation, according to Pulfrey et al. ${ }^{29}$. The temperature of the device was assumed to be $T=300 \mathrm{~K}$, the effective Richardson constant (thermionic emission) $\mathrm{A}^{\star \star}=120 \mathrm{Acm}{ }^{-2} \mathrm{~K}^{-2}$. The black curve shows the maximum power conversion efficiency for the ideal situation in which the barrier height is equal to the band-gap. The black circle indicates the maximum theoretical PCE achievable for a 4-methoxythiophenol (OMeTP) and 4-chlorothiophenol (CITP) modified "a"-MAPbl 3 -"b" bc-PSC. b Contour map of charge collection efficiency as a function of diffusion length $L_{d}$ and electrode separation distance. The dashed lines indicate the current experimental device parameters

However, due to the difference in wettability of anode and cathode ("a" and "b", respectively) the film thickness over electrode "a" is about $240 \mathrm{~nm}$, while over electrode "b" it is $\sim 440 \mathrm{~nm}$ (Supplementary Fig. 10).

KPFM and atomic force microscopy. KPFM and AFM measurements were performed on a Dimension Icon (Veeco) in air. Chromium-platinum coated conductive probes (ElectriMulti75-G, BudgetSensors) were used for the measurements. The scanning area and scanning rate were $25 \times 3.125 \mu \mathrm{m}$ and $0.2 \mathrm{~Hz}$, respectively. The surface roughness has very little influence on the recorded CPD values (Supplementary Fig. 14), whereas the lift height of the AFM tip can greatly affect the magnitude of the CPD (Supplementary Fig. 15). The lift height was 15 $\mathrm{nm}$ for all KPFM measurements.

Raman spectra on fully modified interdigitated electrode. Raman spectroscopy measurements were performed on a Renishaw inVia confocal microscope with a $785 \mathrm{~nm}$ laser operating at $100 \%$ power, in the range $250-1800 \mathrm{~nm}$ with a resolution of $2 \mathrm{~nm}$. Acquisition time was $10 \mathrm{~s}$ per scan with the final spectra from the coaddition of 40 scans. Prior to thiol deposition, gold IDAs were subjected to a surface roughening step via an electrochemical process in order to induce a surfaceenhanced Raman scattering (SERS) effect ${ }^{41}$. In the absence of such a step, the thiols could not be detected on the surface of the IDAs by Raman spectroscopy.

Photoelectron spectroscopy in air. The work functions of thiol modified gold surfaces were investigated using a Riken Keiki Photoelectron Spectrometer (Model AC-2) at $20 \mathrm{nW}$.

Solar cell characterization. The current density-voltage $(J-V)$ characteristics of devices were measured in an inert atmosphere using a Keithley 2400 source meter under $1.5 \mathrm{G}$ light illumination from a solar simulator (Newport $150 \mathrm{~W}$ Xenon lamp coupled with an AM $1.5 \mathrm{G}$ solar spectrum filter). The light intensity was calibrated to $100 \mathrm{~mW} \mathrm{~cm}^{-2}$ using a secondary reference photodiode (Hamamatsu S1133, with KG-5 filter, $2.8 \times 2.4 \mathrm{~mm}$ photosensitive area), which was calibrated by a certified reference cell (PVMeasurements, certified by NREL). The cells were measured from the front side (absorber side) with a back reflector made of $\mathrm{Al}$ foil. For comparison, the $J-V$ characteristics for back side (glass side) illumination were also recorded (Supplementary Fig. 16). The devices were stored in an inert atmosphere and $J-V$ characteristics were obtained after a prolonged storage time (Supplementary Fig. 17). All the $J-V$ characteristics were recorded without a mask since the collected photocurrent originated only from the interdigitated area $(2 \times 2 \mathrm{~mm})$ of the cell (Supplementary Fig. 18).

Photocurrent and photoluminescence mapping. Photocurrent and PL mapping measurements are carried out using a combined detection system, based on a WITec Alpha 300R confocal microscope. A 532-nm laser is fiber-coupled (Thorlabs P5-405BPM-FC-2) through a fiber bench (Thorlabs FBP-A-FC), optically chopped (C995, Terahertz Technologies, Inc., $572 \mathrm{~Hz}$ ) and coupled into the microscope, with the resulting $1 \mu \mathrm{m}$ diameter spot size and $2.8 \mu \mathrm{W}$ power measured at the sample. PL is recorded at short-circuit and summed between 720 and $780 \mathrm{~nm}$, and PC is recorded at short-circuit current using a preamplifier (FEMTO DLPCA-200, Messtechnik GmbH) and a lock-in amplifier (FEMTO LIA-MV-150). The devices were illuminated from the front side (absorber side) and measurements were carried out in an ambient atmosphere with encapsulated devices.
External quantum efficiency. The EQE data were collected using an Oriel $150 \mathrm{~W}$ Xe lamp coupled to a monochromator and an optical fibre in an ambient atmosphere. The devices were illuminated from the front side (absorber side). The light source was chopped at $27 \mathrm{~Hz}$ and the electrical signal was collected under short circuit conditions using a low noise current pre-amplifier (SR570, Stanford Research Systems) and lock-in amplifier (SR830 DSP, Stanford Research Systems). A standard, filtered Si cell from Peccell Limited, which was cross-calibrated with a standard reference cell traceable to the National Renewable Energy Laboratory, was used as the reference. The devices were encapsulated to avoid severe degradation during measurement. However, some gradual degradation of the devices is observed during the measurements despite encapsulation.

Contact angle. Contact angle measurements were obtained using a Contact Angle System OCA (Dataphysics). Milli-Q water was dropped onto samples with a dosing volume of $2 \mu \mathrm{l}$ and a dosing rate of $1 \mu \mathrm{s}^{-1}$.

Scanning electron microscopy and focus ion beam. Cross sections of the samples were prepared by FIB and the SEM cross section images were taken using a FEI Helios NanoLab600 Dual Beam FIB-SEM at a $5 \mathrm{kV}$ accelerating voltage.

Raman spectra of fully modified interdigitated electrodes. Raman spectroscopy confirmed the selective deposition of the functionalized thiols on the finger regions of the gold IDAs (Supplementary Fig. 4), with the Raman spectra of the thiols matching literature values ${ }^{42}$. The fingers of the gold IDAs contained the same characteristic signals of the thiols as the connected bay regions (pad for contact), with the addition of a broad peak centered at $1400 \mathrm{~cm}^{-1}$. This peak can be attributed to photoluminescence of the soda lime glass substrate caused by the 785 $\mathrm{nm}$ laser, which arises from the size of the laser beam being broader than the width of the IDA finger.

The region in which OMeTP was deposited also contained trace amounts of CITP. This is evidenced by a shoulder on the large peak at $1591 \mathrm{~cm}^{-1}$ and a broadening of the base of the peak at $1081 \mathrm{~cm}^{-1}$, which are due to the single peak at $1568 \mathrm{~cm}^{-1}$ and the three peaks centered at $1081 \mathrm{~cm}^{-1}$, respectively, present in the spectrum of pure CITP. This is either due to the partial exchange of OMeTP for CITP on the gold surface during the second thiol deposition step, or CITP depositing onto the gold surface in regions where the OMeTP monolayer deposition was incomplete.

Transfer matrix optical and collection efficiency modelling. Transfer matrix optical modelling of the structure was undertaken in Matlab with bespoke software based on the formalism of Pettersson et al. ${ }^{31}$. The structure was modelled with 70 $\mathrm{nm} \mathrm{Au}$ and $280 \mathrm{~nm}$ perovskite for the contact region (metal-semiconductor interface), and $350 \mathrm{~nm}$ of perovskite over the non-contact region $\left(\mathrm{MAPbI}_{3}\right.$-glass interface). An aluminium reflector was included at the rear of the optically-thick glass substrate, with reflection, absorption and transmission spectra weighted over the contact (width $2.5 \mu \mathrm{m}$ ) and non-contact (width $3.5 \mu \mathrm{m}$ ) regions. Perovskite $n, k$ data were taken from Löper et al., gold from Johnson et al., and aluminium from Rakić et al. ${ }^{43-45}$. A photocurrent of $19.9 \mathrm{~mA} \mathrm{~cm}^{-2}$ was predicted under incident AM $1.5 \mathrm{G}$ solar spectrum, assuming ideal collection efficiency, with greater than $10 \%$ gain expected from the inclusion of a simple quarter-wavelength antireflection coating, consisting of $110 \mathrm{~nm}$ PMMA under a $350 \mathrm{~nm}$ thick perovskite absorber layer ${ }^{46}$. 
The semi-empirical charge carrier diffusion length was obtained by fitting the experimental PC profile (Fig. 4c) using the formalism of Taretto et al. ${ }^{32}$ (Supplementary Fig. 13a). The best fit in respect to peak-to-valley percentage variation of the PC profile (Supplementary Fig. 13b) was chosen to be the $L_{\mathrm{d}}$ value that corresponds to the experimental conditions presented here.

Evaluation of the energy band diagrams for symmetric and asymmetric device constructs at thermal equilibrium in the dark. For the construction of the band diagrams of the symmetric and asymmetric devices, the values of the band gap of $\mathrm{MAPbI}_{3}, E_{\mathrm{g}}=1.6 \mathrm{eV}$, the electron affinity of $\mathrm{MAPbI}_{3}, q \chi_{\text {per }}=3.9 \mathrm{eV}$, and the work function of $\mathrm{MAPbI}_{3}, q \Phi_{\mathrm{s}}=4.7 \mathrm{eV}$, were taken from the literature ${ }^{47}, 48$. The work functions of pristine gold (Au), OMeTP-modified ("a") and ClTP-modified ("b") gold were obtained from the PESA measurements. For the symmetric device $\left(\mathrm{Au}-\mathrm{MAPbI}_{3}-\mathrm{Au}\right)$, the value of the work functions of both the "left", $q \Phi_{\mathrm{m}}^{(\mathrm{a})}$, and "right", $q \Phi_{\mathrm{m}}^{(\mathrm{b})}$, pristine gold contacts was about $4.9 \mathrm{eV}$, whereas in the asymmetric device ("a"-MAPbI ${ }_{3}$ " $\mathrm{b}$ ") the work function values for the OMeTP-modified "left", $q \Phi_{\mathrm{m}}^{(\mathrm{a})}$, and ClTP-modifed "right", $q \Phi_{\mathrm{m}}^{(\mathrm{b})}$, gold contacts were $\sim 4.5 \mathrm{eV}$ and $\sim 5.1 \mathrm{eV}$, respectively.

(1) Symmetric device: In the symmetric Au-MAPbI $3-\mathrm{Au}$ device the band diagram for the metal-semiconductor (M-S) Schottky interface at the "left" contact was the same as for the "right" contact (Supplementary Fig. 5a), and formed backto-back connected Schottky diodes. Therefore, it was sufficient to derive values for the barrier height, $q \Phi_{\mathrm{Bn}}$, and the built-in potential, $q V_{\mathrm{bi}}=q V_{\mathrm{bi}}^{(\mathrm{a})}=q V_{\mathrm{bi}}^{(\mathrm{b})}$, at the $\mathrm{M}-\mathrm{S}$ interfaces for only one of the contacts, i.e. the "left" contact, as follows ${ }^{49,50}$ :

$$
\begin{aligned}
& q \Phi_{\mathrm{Bn}}=q\left(\Phi_{\mathrm{m}}^{(\mathrm{a})}-\chi_{\text {per }}\right)=4.9 \mathrm{eV}-3.9 \mathrm{eV}=1 \mathrm{eV} \\
& q V_{\mathrm{n}}=q\left(\Phi_{\mathrm{s}}-\chi_{\text {per }}\right)=4.7 \mathrm{eV}-3.9 \mathrm{eV}=0.8 \mathrm{eV}
\end{aligned}
$$

$q V_{\mathrm{bi}}=q\left(\Phi_{\mathrm{Bn}}-V_{\mathrm{n}}\right)=q\left(\Phi_{\mathrm{m}}^{(\mathrm{b})}-\Phi_{\mathrm{s}}\right)=0.2 \mathrm{eV}-^{\prime}$ upwards $^{\prime}$ band bending.

For other symmetric devices such as Au-OMeTP-MAPbI ${ }_{3}-\mathrm{OMeTP}-\mathrm{Au}$ and $\mathrm{Au}-$ ClTP-MAPbI 3 -ClTP-Au, the values for $q \Phi_{\mathrm{Bn}}$ and $q V_{\text {bi }}$ can be derived in a similar fashion.

(2) Asymmetric device: In the asymmetric "a"- $\mathrm{MAPbI}_{3}$-"b" device, the S-M Schottky interfaces at the "left" and "right" are not the same, due to different work functions of the "left" and "right" metal contacts $\left(\Phi_{\mathrm{m}}^{(\mathrm{a})} \neq \Phi_{\mathrm{m}}^{(\mathrm{b})}\right)$ (Supplementary Fig. 5b). Therefore, the barrier height and the built-in potential at the respective contacts needed to be considered separately, as follows ${ }^{49,50}$

The "left" contact (Au-OMeTP-MAPbI ${ }_{3}$ :

$$
\begin{aligned}
q \Phi_{\mathrm{Bn}} & =q\left(\Phi_{\mathrm{m}}^{(\mathrm{a})}-\chi_{\text {per }}\right)=4.5 \mathrm{eV}-3.9 \mathrm{eV}=0.6 \mathrm{eV} \\
q V_{\mathrm{n}} & =q\left(\Phi_{\mathrm{s}}-\chi_{\text {per }}\right)=4.7 \mathrm{eV}-3.9 \mathrm{eV}=0.8 \mathrm{eV} \\
q V_{\mathrm{bi}}^{(\mathrm{a})} & =q\left(\Phi_{\mathrm{Bn}}-V_{\mathrm{n}}\right)=q\left(\Phi_{\mathrm{m}}^{(\mathrm{a})}-\Phi_{\mathrm{s}}\right)=0.6 \mathrm{eV}-0.8 \mathrm{eV} \\
& =-0.2 \mathrm{eV}-, \text { downwards, } \mathrm{MAPbI}_{3} \text { energy band bending. }
\end{aligned}
$$

or,

$$
\begin{gathered}
q \Phi_{\mathrm{Bp}}=E_{\mathrm{g}}-q\left(\Phi_{\mathrm{m}}^{(\mathrm{a})}-\chi_{\mathrm{per}}\right)=1.6 \mathrm{eV}-4.5 \mathrm{eV}+3.9 \mathrm{eV}=1 \mathrm{eV} \\
q V_{\mathrm{p}}=E_{\mathrm{g}}-q V_{\mathrm{n}}=E_{\mathrm{g}}-q\left(\Phi_{\mathrm{s}}-\chi_{\mathrm{per}}\right)=1.6 \mathrm{eV}-4.7 \mathrm{eV}+3.9 \mathrm{eV}=0.8 \mathrm{eV} \\
q V_{\mathrm{bi}}^{(\mathrm{a})}=q\left(\Phi_{\mathrm{Bp}}-V_{\mathrm{p}}\right)=1 \mathrm{eV}-0.8 \mathrm{eV} \\
=0.2 \mathrm{eV}-, \text { downwards, } \mathrm{MAPbI}_{3} \text { energy band bending. } \\
E_{\mathrm{g}}=q\left(\Phi_{\mathrm{Bn}}+\Phi_{\mathrm{Bp}}\right)=0.6 \mathrm{eV}+1 \mathrm{eV}=1.6 \mathrm{eV}
\end{gathered}
$$

The "right" contact $\left(\mathrm{MAPbI}_{3}-\mathrm{ClTP}-\mathrm{Au}\right)$ :

$$
\begin{gathered}
q \Phi_{\mathrm{Bn}}=q\left(\Phi_{\mathrm{m}}^{(\mathrm{b})}-\chi_{\text {per }}\right)=5.1 \mathrm{eV}-3.9 \mathrm{eV}=1.2 \mathrm{eV} \\
q V_{\mathrm{n}}=q\left(\Phi_{\mathrm{s}}-\chi_{\mathrm{per}}\right)=4.7 \mathrm{eV}-3.9 \mathrm{eV}=0.8 \mathrm{eV} \\
q V_{\mathrm{bi}}^{(\mathrm{b})}=q\left(\Phi_{\mathrm{Bn}}-V_{\mathrm{n}}\right)=1.2 \mathrm{eV}-0.8 \mathrm{eV} \\
=0.4 \mathrm{eV}-, \text { upwards, } \mathrm{MAPbI}_{3} \text { energy band bending. }
\end{gathered}
$$

Thus, the above calculations suggest that at thermal equilibrium in the dark there is a downwards $\mathrm{MAPbI}_{3}$ energy band bending at the Au-OMeTP-MAPbI M-S interface with a $0.6 \mathrm{eV}$ barrier height, $q \Phi_{\mathrm{Bn}}$, and a $-0.2 \mathrm{eV}$ built-in potential, $V_{\mathrm{bi}}^{(\mathrm{a})}$, whereas at the $\mathrm{MAPbI}_{3}-\mathrm{ClTP}-\mathrm{Au} \mathrm{M}-\mathrm{S}$ interface there is an upwards $\mathrm{MAPbI}_{3}$ energy band bending with a $1.2 \mathrm{eV}$ barrier height and a $0.4 \mathrm{eV}$ built-in potential
(Supplementary Fig. 5b). The band diagram of the asymmetric "a"-MAPbI ${ }_{3}$ " $\mathrm{b}$ " device can be represented as front-to-back connected Schottky diodes.

Theoretical PCE for schottky solar cells. The upper-limit to the maximum theoretical PCE of a cell consisting of a single metal-semiconductor contact (Schottky solar cell) was estimated based on simplified formalism of Pulfrey et al. ${ }^{29}$ by taking into account the reverse current due to thermionic emission over the Schottky barrier in addition to the (short-circuit) photocurrent. The model assumes unit absorbance of all photons with energies above the semiconductor (perovskite) band gap $\left(E_{g}=1.6 \mathrm{eV}\right)$, and ignores all possible non-radiative recombination mechanisms.

The "a"- $\mathrm{MAPbI}_{3}$ contact in "a"-MAPbI $\mathrm{M}_{3}$ "b" and "a"-MAPbI $3-\mathrm{Au}$ device constructs can be considered as an Ohmic contact since the work function of electrode "a" (Au-OMeTP; $4.5 \mathrm{eV})$ is smaller than the work function of the perovskite $(4.7 \mathrm{eV})^{51}$. Thus, this contact does not cause any barrier for electron flow from the semiconductor $\left(\mathrm{MAPbI}_{3}\right)$ to the electrode "a". Work functions of $\mathrm{Au}(4.9 \mathrm{eV})$ and electrode "b" (Au-ClTP; $5.1 \mathrm{eV})$ are larger than the work function of the perovskite $(4.7 \mathrm{eV})$. Therefore, $\mathrm{MAPbI}_{3}-$ " $\mathrm{b}$ " and $\mathrm{MAPbI}_{3}-\mathrm{Au}$ contacts in the "a"-MAPbI ${ }_{3}-\mathrm{Au}$ and "a"-MAPbI 3 " "b" device constructs form the rectifying contacts. Hence, Schottky barriers only at the $\mathrm{MAPbI}_{3}$-"b" $(1.2 \mathrm{eV})$ and $\mathrm{MAPbI}_{3}-\mathrm{Au}(1 \mathrm{eV})$ contacts need to be considered in determining the maximum PCE for the "a"-MAPbI ${ }_{3}^{-}$"b" ( 15\%) and "a"-MAPbI $3-\mathrm{Au}(10 \%)$ devices. In the Au- $\mathrm{MAPbI}_{3}$ " $\mathrm{b}$ " device construct the left $\left(\mathrm{Au}-\mathrm{MAPbI}_{3}\right)$ and right (MAPbI${ }_{3}^{-}$"b") contacts are both rectifying, therefore, the barrier heights for both contacts need to be considered to derive the total current due to thermionic emission over both of the rectifying barriers ${ }^{52}$, and subsequently the PCE of the device.

Data availability. The authors declare that the main data supporting the findings of this study are available within the article and its Supplementary Information files. Extra data are available from the corresponding author upon request.

Received: 4 May 2017 Accepted: 11 July 2017

Published online: 20 September 2017

\section{References}

1. Kojima, A., Teshima, K., Shirai, Y. \& Miyasaka, T. Organometal halide perovskites as visible-light sensitizers for photovoltaic. J. Am. Chem. Soc. 131, 6050-6051 (2009).

2. Lee, M. M., Teuscher, J., Miyasaka, T., Murakami, T. N. \& Snaith, H. J. Efficient hybrid solar cells based on meso-superstructured organometal halide perovskites. Science 338, 643-647 (2012).

3. Burschka, J. et al. Sequential deposition as a route to high-performance perovskite-sensitized solar cells. Nature 499, 316-319 (2013).

4. Yang, W. S. et al. High-performance photovoltaic perovskite layers fabricated through intramolecular exchange. Science 348, 1234-1237 (2015).

5. Lin, Q., Armin, A., Nagiri, R. C. R., Burn, P. L. \& Meredith, P. Electro-optics of perovskite solar cells. Nat. Photon 9, 106-112 (2015).

6. Leijtens, $\mathrm{T}$. et al. Overcoming ultraviolet light instability of sensitized $\mathrm{TiO}_{2}$ with meso-superstructured organometal tri-halide perovskite solar cells. Nat. Commun. 4, 2885 (2013)

7. Unger, E. L. et al. Hysteresis and transient behavior in current-voltage measurements of hybrid-perovskite absorber solar cells. Energy Environ. Sci. 7 , 3690-3698 (2014).

8. Wu, B. et al. Charge accumulation and hysteresis in perovskite-based solar cells: an electro-optical analysis. Adv. Energy Mater. 5, 1500829 (2015).

9. Saidaminov, M. I. et al. Planar-integrated single-crystalline perovskite photodetectors. Nat. Commun. 6, 8724 (2015).

10. Yan, K. et al. High performance graphene-based hole conductor-free perovskite solar cells: Schottky junction enhanced hole extraction and electron blocking. Small 11, 2269-2274 (2015).

11. Chen, L. et al. Organometallic hybrid perovskites: structural, optical characteristic and application in Schottky diode. J. Mater. Sci. Mater. Electron 27, 4275-4280 (2016).

12. Khoram, P., Brittman, S., Dzik, W. I., Reek, J. N. H. \& Garnett, E. C. Growth and characterization of PDMS-stamped halide perovskite single microcrystals. J. Phys. Chem. C 120, 6475-6481 (2016).

13. Xiao, Z. et al. Giant switchable photovoltaic effect in organometal trihalide perovskite devices. Nat. Mater. 14, 193-198 (2015).

14. Zhao, Y. et al. Anomalously large interface charge in polarityswitchable photovoltaic devices: an indication of mobile ions in organic-inorganic halide perovskites. Energy Environ. Sci. 8, 1256-1260 (2015).

15. Yuan, Y. et al. Photovoltaic switching mechanism in lateral structure hybrid perovskite solar cells. Adv. Energy Mater. 5, 1500615 (2015).

16. Dong, Q. et al. Lateral-structure single-crystal hybrid perovskite solar cells via piezoelectric poling. Adv. Mater. 28, 2816-2821 (2016). 
17. Yuan, Y. et al. Electric-field-driven reversible conversion between methylammonium lead triiodide perovskites and lead iodide at elevated temperatures. Adv. Energy Mater. 6, 1501803 (2016).

18. Zhang, Y. et al. Reversible structural swell-shrink and recoverable optical properties in hybrid inorganic-organic perovskite. ACS Nano 10, 7031-7038 (2016).

19. Li, C. et al. Iodine migration and its effect on hysteresis in perovskite solar cells. Adv. Mater. 28, 2446-2454 (2016).

20. Yuan, Y. \& Huang, J. Ion migration in organometal trihalide perovskite and its impact on photovoltaic efficiency and stability. Acc. Chem. Res. 49, 286-293 (2016).

21. Evans, S. D. \& Ulman, A. Surface potential studies of alkyl-thiol monolayers adsorbed on gold. Chem. Phys. Lett. 170, 462-466 (1990).

22. Lü, J. et al. Kelvin probe force microscopy on surfaces: investigation of the surface potential of self-assembled monolayers on gold. Langmuir. 15, 8184-8188 (1999).

23. Alloway, D. M. et al. Interface dipoles arising from self-assembled monolayers on gold: UV-photoemission studies of alkanethiols and partially fluorinated alkanethiols. J. Phys. Chem. B 107, 11690-11699 (2003).

24. Bruening, M. et al. Simultaneous control of surface potential and wetting of solids with chemisorbed multifunctional ligands. J. Am. Chem. Soc. 119, 5720-5728 (1997).

25. de Boer, B., Hadipour, A., Mandoc, M. M., van Woudenbergh, T. \& Blom, P. W. M. Tuning of metal work functions with self-assembled monolayers. Adv. Mater. 17, 621-625 (2005).

26. Liscio, A., Orgiu, E., Mativetsky, J. M., Palermo, V. \& Samorì, P. Bottom-up fabricated asymmetric electrodes for organic electronics. Adv. Mater. 22 5018-5023 (2010).

27. Kobayashi, S. et al. Control of carrier density by self-assembled monolayers in organic field-effect transistors. Nat. Mater. 3, 317-322 (2004).

28. Sum, T. C. \& Mathews, N. Advancements in perovskite solar cells: photophysics behind the photovoltaics. Energy Environ. Sci. 7, 2518-2534 (2014)

29. Pulfrey, D. L. \& McOuat, R. F. Schottky-barrier solar-cell calculations. Appl. Phys. Lett. 24, 167-169 (1974)

30. Grånäs, O., Vinichenko, D. \& Kaxiras, E. Establishing the limits of efficiency of perovskite solar cells from first principles modelling. Sci. Rep. 6, 36108 (2016).

31. Pettersson, L. A. A., Roman, L. S. \& Inganäs, O. Modeling photocurrent action spectra of photovoltaic devices based on organic thin films. J. Appl. Phys. 86, 487-496 (1999).

32. Taretto, K. \& Rau, U. Modeling extremely thin absorber solar cells for optimized design. Prog. Photovolt: Res. Appl. 12, 573-591 (2004).

33. White, T. P., Lal, N. N. \& Catchpole, K. R. Tandem solar cells based on highefficiency c-si bottom cells: top cell requirements for $>30 \%$ efficiency. IEEE $J$. Photovolt 4, 208-214 (2014).

34. Lal, N. N., White, T. P. \& Catchpole, K. R. Optics and light trapping for tandem solar cells on silicon. IEEE J. Photovolt 4, 1380-1386 (2014).

35. Jumabekov, A. N. et al. Back-contacted hybrid organic-inorganic perovskite solar cells. J. Mater. Chem. C 4, 3125-3130 (2016).

36. Santos, A., Deen, M. J. \& Marsal, L. F. Low-cost fabrication technologies for nanostructures: state-of-the-art and potential. Nanotechnology 26, 042001 (2015).

37. Huang, F. et al. Gas-assisted preparation of lead iodide perovskite films consisting of a monolayer of single crystalline grains for high efficiency planar solar cells. Nano Energy 10, 10-18 (2014).

38. Bard, A. J. \& Faulkner, L. R. Electrochemical Methods: Fundamentals and Applications edn 2 (Wiley, New York, 2000).

39. Strutwolf, J. \& O'Sullivan, C. K. Microstructures by selective desorption of selfassembled monolayer from polycrystalline gold electrodes. Electroanalysis 19 1467-1475 (2007)

40. Henry, O. Y. F., Maliszewska, A. \& O'Sullivan, C. K. DNA surface nanopatterning by selective reductive desorption from polycrystalline gold electrode. Electrochem. Commun. 11, 664-667 (2009).

41. Michota, A. \& Bukowska, J. Surface-enhanced Raman scattering (SERS) of 4-mercaptobenzoic acid on silver and gold substrates. J. Raman Spectrosc. 34, $21-25$ (2003)

42. Kang, H. et al. Direct identification of on-bead peptides using surface-enhanced raman spectroscopic barcoding system for high-throughput bioanalysis. Sci. Rep. 5, 10144 (2015).

43. Löper, F. et al. Complex refractive index spectra of $\mathrm{CH}_{3} \mathrm{NH}_{3} \mathrm{PbI}_{3}$ perovskite thin films determined by spectroscopic ellipsometry and spectrophotometry. J. Phys. Chem. Lett. 6, 66-71 (2015).

44. Johnson, P. B. \& Christy, R. W. Optical constants of the noble metals. Phys. Rev. B 6, 4370-4379 (1972)
45. Rakić, A. D., Djurišić, A. B., Elazar, J. M. \& Majewski, M. L. Optical properties of metallic films for vertical-cavity optoelectronic devices. Appl. Opt. 37, 5271-5283 (1998).

46. Beadie, G., Brindza, M., Flynn, R. A., Rosenberg, A. \& Shirk, J. S. Refractive index measurements of poly(methyl methacrylate) (PMMA) from 0.4-1.6 $\mu \mathrm{m}$. Appl. Opt. 54, F139-F143 (2015).

47. Wang, C. et al. Surface analytical investigation on organometal triiodide perovskite. J. Vac. Sci. Technol. B 33, 032401 (2015).

48. Liu, X. et al. Electronic structures at the interface between $\mathrm{Au}$ and $\mathrm{CH}_{3} \mathrm{NH}_{3} \mathrm{PbI}_{3}$. Phys. Chem. Chem. Phys. 17, 896-902 (2015).

49. Sze, S. M. \& Ng, K. K. Physics of Semiconductor Devices edn 3 (Wiley-Interscience, Hoboken, 2007).

50. Sze, S. M., Coleman, D. J. \& Loya, A. Jr. Current transport in metalsemiconductor-metal (MSM) structures. Solid-St. Electron 14, 1209-1218 (1971).

51. Rhoderick, E. H. \& Williams, R. H. Metal-Semiconductor Contacts edn 2 (Clarendon Press, Oxford, 1988).

52. Osvald, J. Back-to-back connected asymmetric Schottky diodes with series resistance as a single diode. Phys. Status Solidi A 212, 2754-2758 (2015).

\section{Acknowledgements}

This work was performed in part at the Melbourne Centre for Nanofabrication (MCN) in the Victorian Node of the Australian National Fabrication Facility (ANFF). The authors thank Dr Alexandr Simonov, Mr Dijon Hoogeveen and Dr Shannon A. Bonke for their help with electrochemistry experiments, as well as Dr Soon Hock Ng and Dr Julian Lloyd for their help with the design of the graphs. This work was funded through CSIRO Manufacturing as part of an Office of the Chief Executive Postdoctoral Fellowship (A.N.J.) and Science Leader Fellowship (U.B.). We also acknowledge the financial support from the Australian Research Council (project number DP160104575), the Australian Renewable Energy Agency (ARENA) and the Australian Centre for Advanced Photovoltaics (ACAP). D.E.G. acknowledges the ARC for support through a Future Fellowship (FT140100514). Q. Bao acknowledges the support from the Australian Research Council (ARC) Future Fellowship (project number: FT150100450) and Centre of Excellence in Future Low-Energy Electronics Technologies (FLEET) (project number CE170100039).

\section{Author contributions}

X.L., A.N.J. and U.B.: Designed and directed the study. A.R.P. and Y.C.: Deposited perovskite absorber layer. N.N.L., Y.Z. and Q.B.: Carried out the photocurrent and photoluminescence mapping measurements and analysis. D.E.G., N.W.D.: Carried out theoretical calculations. A.S.R.C.: Carried out Raman spectroscopy measurements and analysis. K.S.: Carried out the external quantum efficiency measurements and analysis. M.F. and L.S.: Performed the electrochemical desorption experiments. X.L., A.N.J. and U.B.: Wrote the manuscript. All authors commented on the paper.

\section{Additional information}

Supplementary Information accompanies this paper at doi:10.1038/s41467-017-00588-3

Competing interests: The authors declare no competing financial interests.

Reprints and permission information is available online at http://npg.nature.com/ reprintsandpermissions/

Publisher's note: Springer Nature remains neutral with regard to jurisdictional claims in published maps and institutional affiliations.

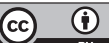

Open Access This article is licensed under a Creative Commons Attribution 4.0 International License, which permits use, sharing, adaptation, distribution and reproduction in any medium or format, as long as you give appropriate credit to the original author(s) and the source, provide a link to the Creative Commons license, and indicate if changes were made. The images or other third party material in this article are included in the article's Creative Commons license, unless indicated otherwise in a credit line to the material. If material is not included in the article's Creative Commons license and your intended use is not permitted by statutory regulation or exceeds the permitted use, you will need to obtain permission directly from the copyright holder. To view a copy of this license, visit http://creativecommons.org/ licenses/by/4.0/.

(C) The Author(s) 2017 


\section{University Library}

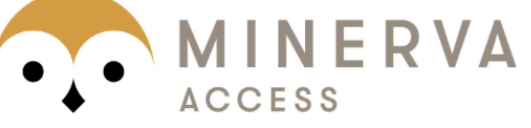

A gateway to Melbourne's research publications

Minerva Access is the Institutional Repository of The University of Melbourne

Author/s:

Lin, X;Jumabekov, AN;Lal, NN;Pascoe, AR;Gomez, DE;Duffy, NW;Chesman, ASR;Sears,

K;Fournier, M;Zhang, Y;Bao, Q;Cheng, Y-B;Spiccia, L;Bach, U

Title:

Dipole-field-assisted charge extraction in metal-perovskite-metal back-contact solar cells

Date:

2017-09-20

Citation:

Lin, X., Jumabekov, A. N., Lal, N. N., Pascoe, A. R., Gomez, D. E., Duffy, N. W., Chesman, A. S. R., Sears, K., Fournier, M., Zhang, Y., Bao, Q., Cheng, Y. -B., Spiccia, L. \& Bach, U. (2017). Dipole-field-assisted charge extraction in metal-perovskite-metal back-contact solar cells. NATURE COMMUNICATIONS, 8 (1), https://doi.org/10.1038/s41467-017-00588-3.

Persistent Link:

http://hdl.handle.net/11343/255101

License:

CC BY 